\title{
MBE Growth Of GaN Films In Presence Of Surfactants: The Effect Of Mg And Si
}

\author{
Guido Mula ${ }^{1,2}$, Bruno Daudin ${ }^{2}$, Christoph Adelmann' ${ }^{2}$, Philippe Peyla ${ }^{3}$ \\ ${ }^{1}$ INFM and Dipartimento di Fisica, Università di Cagliari, Cittadella Universitaria, \\ Strada Prov.le Monserrato - Sestu Km 0.700, 09042 Monserrato (CA), Italy. \\ ${ }^{2}$ Département de Recherche Fondamentale sur la Matière Condensée - SP2M - \\ Laboratoire de Physique des Semiconducteurs, CEA-Grenoble, 17, Rue des Martyrs, \\ 38054 Grenoble cedex 9, France \\ ${ }^{3}$ Laboratoire de Physique et Modélisation des Milieux Condensés, Maison des \\ Magistères, Université Joseph Fourier-CNRS, BP 166, 38042 Grenoble cedex 9, France.
}

\section{ABSTRACT}

We present here a description and an analysis of the modifications in the growth behaviour of $\mathrm{GaN}$ induced by the presence of foreign species. The particular cases of $\mathrm{Mg}$ and $\mathrm{Si}$ are analysed. Profound changes, both in microscopic and macroscopic scales, occur in presence of $\mathrm{Mg}$, even for fluxes of about $1 / 1000^{\text {th }}$ of the Ga flux. The growth rate can be increased by almost $50 \%$, depending of the III/V ratio and on the amount of $\mathrm{Mg}$. A theoretical model is proposed to describe the observed effect. It is found that $\mathrm{Mg}$ induces changes in the $\mathrm{Ga}$ and $\mathrm{N}$ diffusion barriers and acts as a surfactant. The effect is stronger on the $\alpha-\mathrm{GaN}$ than on the $\beta-\mathrm{GaN}$, where $\mathrm{N}$ is more tightly bonded. The effect of $\mathrm{Si}$ is by far less pronounced, probably because it is more easily incorporated than $\mathrm{Mg}$, and its effect on the surface kinetics is then strongly reduced.

\section{INTRODUCTION}

The understanding of the kinetics of the growing species on the GaN surface is of paramount interest for optimal epitaxial growth. In particular, the growth by Molecular Beam Epitaxy (MBE) is still far from perfection, partially due to the strong dependence of the surface morphology on the III/V ratio. This situation is especially true for plasmasource MBE, where the growth in Ga-rich regime leads to a metal accumulation that eventually blocks the growth process, while the N-rich regime leads to rough surfaces. Therefore, the use of surfactants to modify the surface mobility of the chemical species can be a useful step in the search for optimisation. In fact, the determination of less critical growth conditions, where the surface can be flat and free of Ga droplets, should lead to better optical and structural properties.

A few possible candidates for surfactants have already been examined. Theoretical [1] and experimental [2] reports show that As presence on the $\beta-\mathrm{GaN}$ surface leads to preferential growth of the cubic phase and to flatter surfaces. The use of $\mathrm{Mg}$ and $\mathrm{Si}$ during metal-organic chemical vapour deposition (MOCVD) growth [3] is shown to improve structural quality by reducing the defect density. The addition of In also shows significant surfactant effect [4], increasing the surface mobility. Si seems to have an "anti-surfactant" effect on MOCVD-grown $\alpha-G a N$ [5]. What can be extracted from these results is that the $\mathrm{GaN}$ growth seems to be extremely dependent on the presence of foreign species. $\mathrm{Mg}$ and $\mathrm{Si}$ are the most commonly used materials for doping, and the knowledge of their influence on the growth parameter is clearly fundamental. We will 
present here a study on $\mathrm{Mg}$ - and Si-induced modifications of the growth kinetics at $\alpha$ and $\beta-\mathrm{GaN}$ surfaces. A phenomenological model is proposed for the $\alpha-\mathrm{GaN}$ phase.

\section{EXPERIMENTAL DETAILS}

All samples were grown by plasma-assisted molecular beam epitaxy (MBE) in a MECA2000 growth chamber using an EPI Unibulb source for nitrogen. The typical $\mathrm{N}_{2}$ flow was about $0.5 \mathrm{sccm}$ for a forward power of $300 \mathrm{~W}$. The growth temperature, read by a thermocouple on the sample backside, ranged from 650 to $680^{\circ} \mathrm{C}$. The substrates were both Metal-Organic Chemical Vapour Deposition (MOCVD) epiready GaN/sapphire or sapphire (0001)-oriented for $\alpha-\mathrm{GaN}$. For the $\beta-\mathrm{GaN}$ the substrates were (001)-oriented $\mathrm{SiC}$ deposited by CVD on (001)-oriented Si substrates. The samples were analysed in situ by Reflection High-Energy Electron Diffraction (RHEED) and ex-situ by optical microscopy (OM). The OM images were used to analyse the Ga-droplet distribution.

\section{RESULTS AND DISCUSSION}

\section{The effect of Mg - $\alpha$-GaN growth}

Here the observed effects are by far more striking and interesting. Figure 1 shows the growth rate of $\alpha-\mathrm{GaN}$ as a function of the Ga cell temperature $\mathrm{T}_{\mathrm{Ga}}$ for a given $\mathrm{N}$ plasma condition and for different impinging Mg fluxes. The reference curve obtained without $\mathrm{Mg}$ is also shown for comparison. The growth rates have been determined by RHEED oscillations. Eye-guides are added for an easier analysis. The "residual surface $\mathrm{Mg}$ " label refers to $\mathrm{Mg}$ atoms desorbed from the growth chamber walls in the few days following the use of the $\mathrm{Mg}$ cell, due to the high $\mathrm{Mg}$ vapour pressure. It is difficult to precisely evaluate this flux, but a reasonable estimate sets it to a few orders of magnitude lower than a standard doping flux. A first remarkable feature in figure 1 is the overall increase in growth rate observed using a very low $\mathrm{Mg}$ flux. This increase can be as high as about $40 \%$ in the case of the curve obtained with a $\mathrm{Mg}$ cell temperature of $230^{\circ} \mathrm{C}$. It is important to stress that the maximum $\mathrm{Mg}$ flux used here is of the order of $1 / 1000^{\text {th }}$ of the Ga flux. This implies that is impossible to explain the growth rate increase by the simple

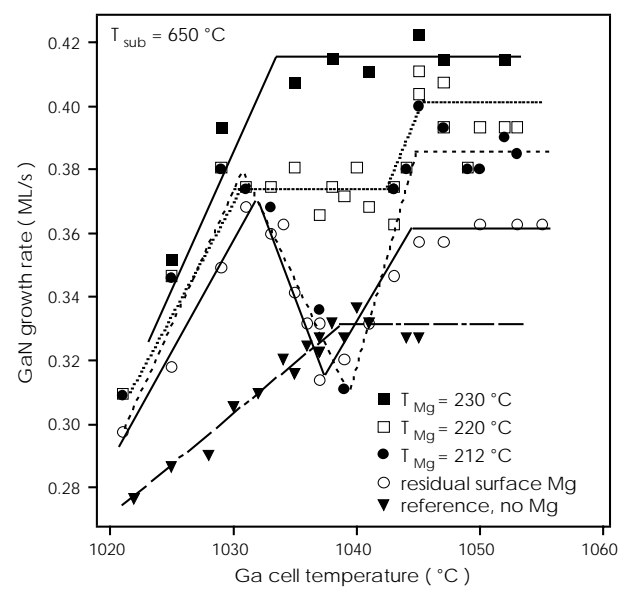
incorporation of $\mathrm{Mg}$ into the $\mathrm{GaN}$ matrix. The effect must then be related to significant $\mathrm{Mg}$-induced changes in the surface kinetics. Another even more striking feature is the very surprising "hollow" in the growth rate observed for low Mg fluxes: above a critical Ga flux there is a very sharp decrease in the growth rate, followed by a new increase at still higher Ga

Figure 1. $\alpha-\mathrm{GaN}$ growth rate as a function of the Ga cell temperature for various $\mathrm{Mg}$ cell temperatures. Eyeguides are added for an easier reading. 


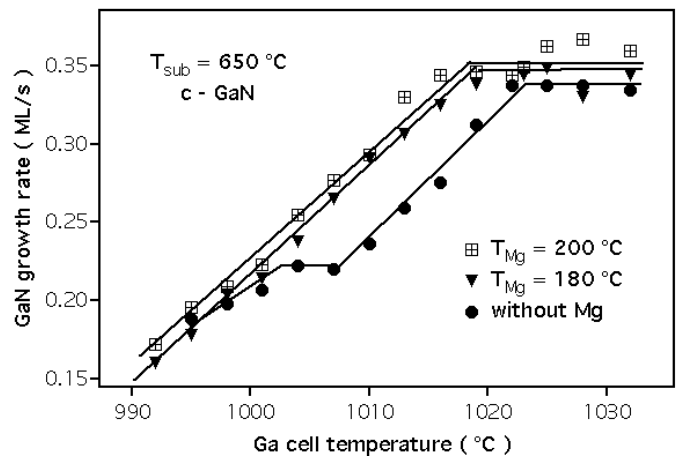

Figure 2. $\beta-\mathrm{GaN}$ growth rate as a function of $\mathrm{T}_{\mathrm{Ga}}$ and for different temperatures of the $\mathrm{Mg}$ cell. The substrate temperature is $650^{\circ} \mathrm{C}$.

fluxes. A more detailed analysis reveals that the growth rate slope in the Ga-limited regime is steeper in presence of $\mathrm{Mg}$ than without it, and that the new slope is in better agreement with the one expected from the Ga-cell flux

behavior. For higher $\mathrm{Mg}$ cell temperatures $\left(\mathrm{T}_{\mathrm{Mg}}\right)$, the hollow smoothens and eventually disappears. Above $\mathrm{T}_{\mathrm{Mg}}=230^{\circ} \mathrm{C}$ there was no further modification of the growth rate. Moreover, the RHEED pattern starts to degrade, indicating a rougher surface, and the RHEED oscillations were very difficult to observe. This behavior seems to be quite independent on the growth parameters. We do not observe qualitative changes increasing the $\mathrm{N}$ flow by a factor of 2 and/or varying the substrate temperature $\left(650-680^{\circ} \mathrm{C}\right)$.

As far as the RHEED pattern is concerned, the most surprising effect is that the pattern remains streaky for $\mathrm{Ga} / \mathrm{N}$ ratios quite far in the $\mathrm{N}$-rich regime (the growth rate is decreased by almost 30\%). The RHEED oscillations were intense and persistent below the critical $\mathrm{T}_{\mathrm{Ga}}$ (from four-five clear oscillations for the lowest $\mathrm{Ga}$ flux to few tens near the critical point), slightly poorer within the hollow, where just 4-5 oscillations where visible, and improved again when further increasing the Ga flux. This seems to indicate two main phenomena: the first is that the surface seems to stay flat for growth in the Galimited regime, leading to a Ga droplets-free growth. The second is that something dramatic is happening in the vicinity of the critical Ga flux value, affecting the mobility of the species on the surface. These results will be discussed in more detail later in this article, when all the experimental details will be presented.

\section{The effect of $\mathrm{Mg}-\beta$-GaN growth}

The growth of the cubic GaN phase is less affected by the presence of $\mathrm{Mg}$ than the hexagonal phase. In figure 2 we show the dependence of the growth rate on the Ga flux in the case of $\beta-\mathrm{GaN}$, for two different impinging $\mathrm{Mg}$ fluxes. The reference curve without $\mathrm{Mg}$ is also shown for comparison. The observed behaviour is quite different from the $\alpha$ $\mathrm{GaN}$ case. There is no longer a hollow, and the growth rate curve is quite regular. Adding $\mathrm{Mg}$, the plateau around $\mathrm{T}_{\mathrm{Ga}}=1005^{\circ} \mathrm{C}$ disappears, the growth rate at higher $\mathrm{T}_{\mathrm{Ga}}$ is increased, and no appreciable difference in the slope or in the final growth rate can be observed. Within the $\mathrm{T}_{\mathrm{Mg}}$ range explored, the effect of $\mathrm{Mg}$ seems to be independent on the $\mathrm{Mg}$ flux value.

\section{The effect of Si}

There is a main difference in the behavior of $\mathrm{Si}$ and $\mathrm{Mg}$ atoms on the surface of $\mathrm{GaN}$. Within the flux range we explored (doping level), the Si atoms are easily 
incorporated in the $\mathrm{GaN}$ matrix, limiting as a consequence the surface effect. As a matter of fact, to observe any effect on the kinetics of the Ga and $\mathrm{N}$ species we needed to create an accumulation of $\mathrm{Si}$ atoms on the surface, exposing the $\mathrm{GaN}$ surface to $\mathrm{Si}$ alone for a few seconds before starting the growth. The only observable effect was an increase of the growth rate in the plateau region as if we were not in the $\mathrm{N}$-saturated regime. This is another clear indication, consistent with the results obtained with $\mathrm{Mg}$, that the incorporation coefficient of $\mathrm{N}$ is definitely not unity. We tentatively attribute this behavior to a longer permanence time of $\mathrm{N}$ atoms on the surface, easing then their incorporation by the formation of $\mathrm{GaN}$.

\section{Optical microscopy and discussion}

The observation of the hollow in the growth rate of $\alpha-\mathrm{GaN}$ is quite a surprising phenomenon. How can a growth rate decrease when the amount of the available species increases? Before answering the question, a premise must be done about the reliability of RHEED oscillations for the measurement of growth rates. Several papers in the literature [6] point out that RHEED oscillations may be unreliable for determining growth rates, as in the case of step flow or bilayer growth. In our case, apart from the hollow, we observe a huge increase of the growth rate, ruling out the step flow argument. The regular variation of the growth rate excludes a possible frequency doubling effect. As far as the hollow is concerned, its appearance is so sharp that is difficult to believe it an artifact. In the following paragraphs we will discuss the possible meanings of the hollow, explaining at the same time why we strongly believe that this hollow corresponds to reality.

To understand the hollow, it is important to observe the full behavior of the growth rate, and in particular the fact that the slope of the curve in the Ga-limited regime is steeper in presence of $\mathrm{Mg}$. It is well known that the excess $\mathrm{Ga}$ at the surface leads to the formation of Ga droplets, and it is a reasonable assumption that near the stoichiometry there are surface portions where the formation of Ga droplets becomes possible. This implies that the formation of $\mathrm{Ga}$ droplets should be also possible in the slightly N-rich regime. If we suppose that the presence of $\mathrm{Mg}$ on the surface stops the formation of $\mathrm{Ga}$ droplets, the slope of the growth rate should increase, because more $\mathrm{Ga}$ atoms would be available for the growth. The hollow in the growth rate can then be tentatively attributed to the sudden formation of Ga droplets above a critical Ga flux. To check our hypothesis, we performed OM measurements on the surface of $\alpha-\mathrm{GaN}$ samples grown with and without $\mathrm{Mg}$. The growth geometry was adapted to have a strong $\mathrm{Ga}$ flux gradient along the surface, so to be able to directly observe the onset of the Ga droplets formation as a function of the Ga flux. The Ga flux gradient was as high as $10 \% / \mathrm{cm}$. In figure 3 are shown two OM micrographs of the surface of samples without (left) and with (right) $\mathrm{Mg}$. Below each micrograph there is the plot of the corresponding droplet counts over subsequent slices $0.1 \mathrm{~mm}$ wide and $1 \mathrm{~mm}$ high. It appears clearly from the comparison of these two pictures that the threshold for Ga droplets formation is much sharper in presence of $\mathrm{Mg}$ than without it. On $\beta-\mathrm{GaN}$ (not shown), the droplet distribution with and without $\mathrm{Mg}$ is almost identical to the one observed for $\alpha-\mathrm{GaN}$ without $\mathrm{Mg}$.

We developed a phenomenological model for $\alpha-\mathrm{GaN}$, described in more detail in the reference [7], that takes into account in a simple way the Ga atoms capture cross sections for the formation of Ga droplets and for the incorporation into the GaN matrix. No specific assumptions are made on $\mathrm{N}$, which is supposed to be available all the time. This means that our model is valid in the N-rich regime. Should the N sticking coefficient 

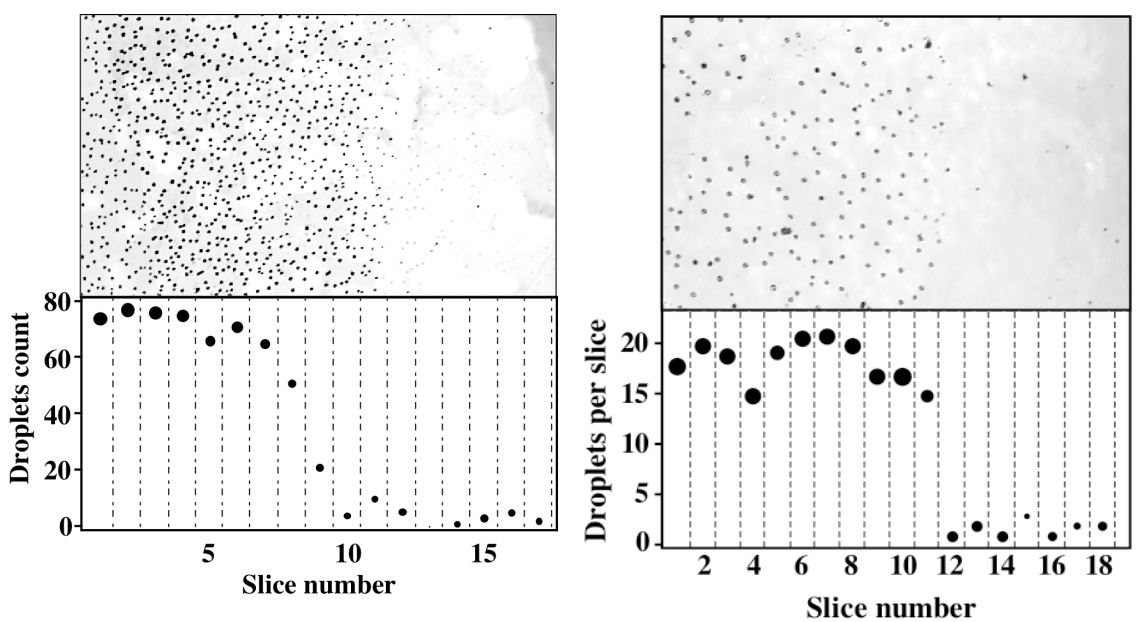

Figure 3. Optical micrographs (transmission mode) of the surface of $\alpha-\mathrm{GaN}$ layers grown without (left) and with (right) $\mathrm{Mg}$. The Ga flux gradient is about $10 \% / \mathrm{cm}$ (right to left). Below each micrograph there is a plot of the corresponding droplet counting over slices $1 \mathrm{~mm}$ wide and $0.1 \mathrm{~mm}$ high. The slice number is reported on the $\mathrm{x}$-axis. The micrographs represent the measured area. The size of the markers is representative of the droplet size.
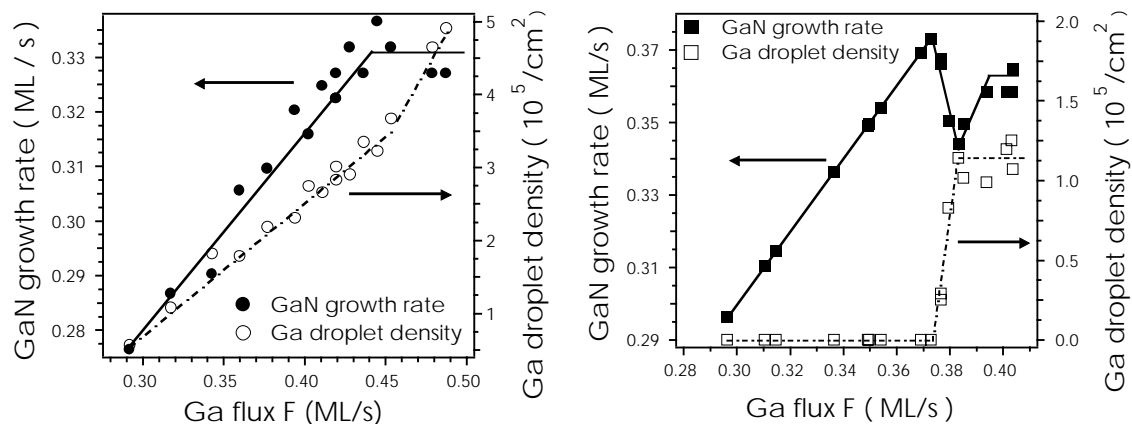

Figure 4. Growth rates (full symbols, left axis) for $\alpha-\mathrm{GaN}$ without (left) and with (right) $\mathrm{Mg}$. The corresponding Ga droplet densities (open symbols, left axis), obtained using our model, are also shown.

be lower than one, the number of available $\mathrm{N}$ atoms will be larger than the amount actually used, and our model will hold also for the Ga rich regime. We will not further discuss these issues here. In figure 4 we show the experimental growth rates (full symbols, left axis) obtained for $\alpha-G a N$ without (left) and with (right) Mg. The open symbols represent the corresponding Ga droplet density (right axis) obtained with our model through a fit to the experimental data. If we compare these results with those represented in the figure 3 we cannot but notice their remarkable agreement. First of all, the model explains the hollow with a sharp onset of the formation of Ga droplets, exactly 
as we observed experimentally. Second, the ratio of the droplet densities, with and without $\mathrm{Mg}$, is about four in both the measurements and the theoretical fit.

These results clearly suggest that the Mg-induced kinetics modifications are a reduction of the $\mathrm{Ga}$ atoms mobility in the Ga-limited region (no droplets can be formed) and an increase of the mobility in the Ga-rich regime (the droplets are larger and less dense). Moreover, the amount of $\mathrm{N}$ available for the growth seems to be larger than what is usually assumed: the presence of $\mathrm{Mg}$ allows for a growth rate increase as high as about $30 \%$. This strongly suggests that the sticking coefficient of $\mathrm{N}$ is not one and that it is highly dependent on the surface kinetics.

The effects on the $\beta-\mathrm{GaN}$ surface are considerably less pronounced, although the presence of $\mathrm{Mg}$ seems again to stop the Ga-droplet formation in the Ga-limited regime. As a matter of fact, we saw in figure 2 that the presence of $\mathrm{Mg}$ cancels the step in the growth rate present in the Ga-limited regime without $\mathrm{Mg}$. The step is very reasonably related to the onset of Ga-droplet formation in the Ga-limited regime. This onset being very smooth, compared to the hollow of the $\alpha-\mathrm{GaN}$, it is very difficult to see the difference by means of optical microscopy of the growth with and without $\mathrm{Mg}$.

\section{CONCLUSIONS}

The present study shows the deep changes induced by the presence of $\mathrm{Mg}$ and $\mathrm{Si}$ on the GaN surfaces during growth. Our experiments clearly indicate that the surface mobility on $\alpha-\mathrm{GaN}$ can be dramatically affected by the presence of $\mathrm{Mg}$. Moreover, several details strongly indicate that the sticking coefficient of $\mathrm{N}$ is probably far from unity and that more detailed studies on the MBE growth of GaN are necessary for improving samples quality.

\section{REFERENCES}

1. T. Zywietz, Jörg Neugebauer, M. Scheffler, J. Northrup, C.G.Van de Walle, MRS Internet J. of Nitride Semicond. Res., 3, Article 26, (1998).

2. H. Okumura, H. Hamaguchi, G. Feuillet, Y. Ishida, S. Yoshida, Appl. Phys. Lett., 72, 3056 (1998); G. Feuillet, H. Hamaguchi, K. Ohta, P. Hacke, H. Okumura, S. Yoshida, Appl. Phys. Lett., 70, 1025 (1997).

3. B. Beaumont, S. Haffouz, P. Gibart, Appl. Phys. Lett., 72, 921 (1998); S. Haffouz, B. Beaumont, P. Gibart, MRS Internet J. of Nitride Semicond. Res., 2, Article 8 (1998).

4. F. Widmann, B. Daudin, G. Feuillet, N. Pelekanos, J.L. Rouvière, Appl. Phys. Lett., 73, 2642 (1998).

5. H. Hirayama, Y. Aoyagi, S.Tanaka, MRS Internet J. Nitride Semicond. Res., 4S1, G9.4 (1999).

6. E.S. Tok, J.H. Neave, F.E. Allegretti, J. Zhang, T.S. Jones, B.A. Joyce, Surf. Sci. 371, 277 (1997); K. Sato, M.R. Fahy, B.A. Joyce, Surf. Sci. 315, 105 (1994).

7. Guido Mula, B. Daudin, P. Peyla, Proceedings of the $3^{\text {rd }}$ Int. Conference on Nitride Semiconductors, Montpellier (France), July 1999, in press. 